\title{
Improvement of sensor response reproducibility and multistage recognition approach for samples with dominant components
}

\author{
I.V. Kruglenko \\ V. Lashkaryov Institute of Semiconductor Physics, NAS Ukraine \\ 41 Prospect Nauky, Kyiv 03028, Ukraine \\ Tel.: (380-44) 525-56-26; Fax: (380-44) 525-83-42; e-mail: kruglenko@yahoo.com
}

\begin{abstract}
A multistage recognition approach is advanced for poorly classified clusters. According to this approach, if a sample is related to a cluster that is common for several samples, then further object recognition (within that cluster) is possible. Such two-stage recognition procedure is based, at each stage, on the fuzzy logic concept and enables one to perform practically complete recognition of all the samples under consideration.
\end{abstract}

Keywords: "Electronic Nose", dominant components, multistage recognition procedure.

Manuscript received 00.00.00; accepted for publication 00.00.00; published online 00.00.08.

\section{Introduction}

The smart sensor systems are important facilities applied when dealing with provision of efficiency and environmental safety of industrial production, as well as improvement of life quality [1].One of the problems occurring in sensor devices is temporal variation of sensitivity and selectivity profile of sensitive elements. Such drifts affect image recognition by limiting recognition ability of the system used, thus requiring additional gauging. In some cases, however, it is possible to avoid sensor surface modification in the course of measurements by using a specific procedure of surface treatment. Indeed, just the sensitive layers determine almost all limitations of "Electronic Nose" stability. Therefore, stability of sensor operation during its service life involves stability and reproducibility of the sensitive layer parameters [2].

A possibility to choose specific unique selectivity profiles out of various molecular organic materials makes them promising for application as sensitive coatings in multichannel analyzers. The organic molecules can interact with a wide range of various analytes. This means also prevalent effect of dominant components in complex mixtures, such as drinks $[3,4]$, perfumes [5] and pharmaceuticals [6,7], on sensor response. Two different types of sensor-gas mixture interaction may form the basis of the above process, namely, (i) gas molecules interact only with the receptor centers at the film surface or in the layer bulk; (ii) sorption occurs in the lattice voids and is not related directly to the receptor-analyte interaction; these two processes lead to variation of the layer structure.
Obviously, the above two processes can result in long-term relaxation. Indeed, the investigations with surface plasmon resonance and ellipsometry techniques [8] (that were confirmed with the results of atomic force microscopy studies) showed that both topography and thickness of calixarenes sensitive layers varied due to adsorption of organic molecules, such as toluene, chloroform, and ethanol [9]. These variations depended on the gas nature and time of exposure.

To illustrate, the thickness of thin C[4]A layers increased by $12 \%$ at the initial stage of sorption (Fig. 1). After exposure to chloroform vapor for over 30 min., the layer thickness increase was $6 \div 8 \%$ the initial thickness value. The above process seems to be due to film structure variation because of solid structure relaxation.

The atomic force microscopy studies showed that topography of thin $(\approx 100 \mathrm{~nm})$ calixarenes layers changed (see Fig. 2) after their surface was exposed to saturated vapors of solvents [10]. Thus, in some cases, interaction of thin organic layers with solvent vapors leads to undesired processes (from the viewpoint of stability of the gas sensor parameters). It is obvious that the properties of such layers in what concerns their interaction with different gaseous analytes will vary.

\section{Experimental procedure}

A fully automated quartz crystal microbalance set designed at ISP, Kyiv (8 channels, $10 \mathrm{MHz}$ AT-cut crystals) with a measurement interval of $1 \mathrm{~s}$ was used in our experiments [7]. The measuring procedure involved the following stages: argon circulation; brandy vapor-argon mixture circulation (bubbling, $12 \mathrm{ml}$ of 


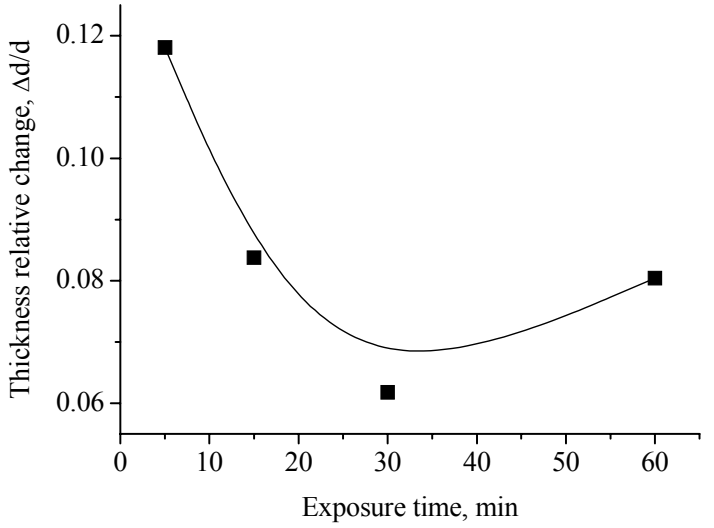

Fig. 1. Variation of thickness of deposited C[4]A layers with time of exposure to chloroform vapor (layer thickness of $60-70 \mathrm{~nm})$.

probe, rate $50 \mathrm{ml} / \mathrm{min}$, and temperature of $36 \pm 0.3{ }^{\circ} \mathrm{C}$ ); argon circulation; ethanol-argon mixture circulation, if any. Thin films of C[n]A (tret-butylcalyx[n] arene, $\mathrm{n}=4$, 6 , 8), tetracene, pentacene, phtalocyanine $\left(\mathrm{H}_{2} \mathrm{Pc}\right)$, dibenzotetraazaannulene $\left(\mathrm{H}_{2}\right.$ TAA $)$ and tetramethyldibenzotetraazaannulene ( $\mathrm{H}_{2}$ TMTAA) were prepared by thermal sputtering onto the metal electrodes of QCM plates [11]. Thin films (about $100 \mathrm{~nm}$ ) were prepared by thermal sputtering in vacuum (VUP-5M, pressure of $5 \times 10^{-4} \mathrm{~Pa}$, temperature of $297 \pm 2 \mathrm{~K}$, deposition rate of $0.1 \mathrm{~nm} / \mathrm{min}$ ).

Five different samples were studied, namely, those of ethanol and four brandy sorts (State Standard ГOCT13741) produced by Public Corporation APF "Tavriya" (Nova Kakhovka, Ukraine): "Borysphen", "Georgiyivs'kyi", "Oleksandriys'kyi" and "Tavriya" (from here on $\mathrm{B}, \mathrm{G}, \mathrm{O}$, and $\mathrm{T}$, respectively).

Restoration of sensitive layers using ethanol vapor. If several (three-four) experiments with vapors of drinks (e.g. brandy) having volatile organic components were performed in succession, then irreproducibility of the results of measurements due to surface contamination was observed It is obvious that image recognition in such situation is impossible. Indeed, a comparative analysis showed that direct measurements of brandy samples without additional treatment did not give unambiguous solution to the problem of chemical image recognition.

Bearing in mind that the results obtained could be due to incomplete surface cleaning as well as surface modification, we tested the following restoration procedure for sensitive coating parameters. Between the successive experiments with drink samples, the sensors were cleaned by exposing to gas-vapor mixture of saturated ethanol vapor and argon during $5 \mathrm{~min}$. It was supposed that this procedure could ensure not only removal of organic molecules from the sensitive layer but restoration of the surface initial state as well. We also made check measurements without sensor surface treatment with vapor.
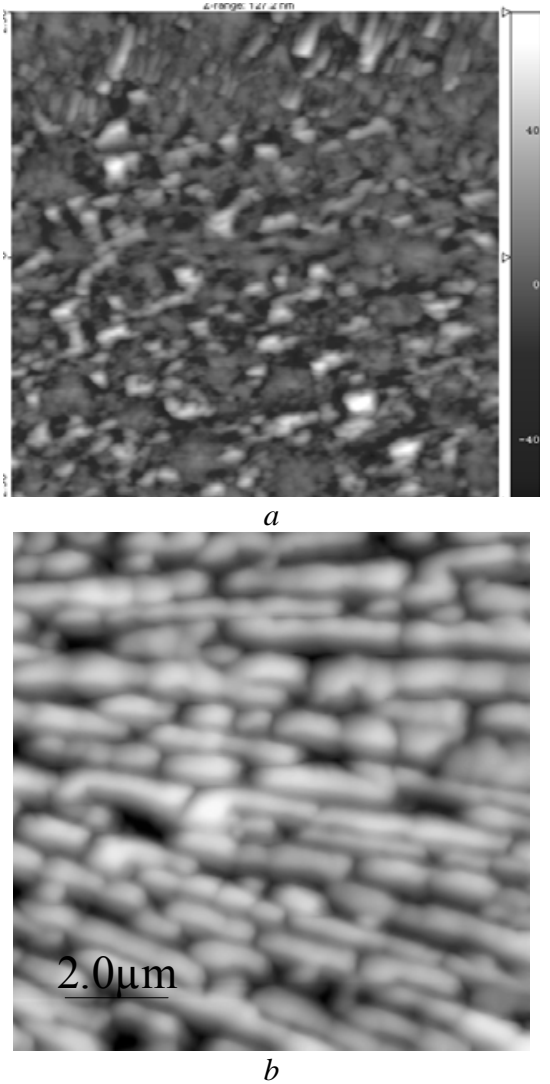

Fig. 2. Topography of calixarene C[4]A surfaces before (left) and after (right) treatment in chloroform vapor.

An analysis of chemical images obtained demonstrated complete coincidence of the images for three successive experiments (Fig. 3). One can see that a simple procedure of sensor array treatment with ethanol vapor can improve substantially system restoration thus ensuring formation of stable chemical images [12].

"Electronic Nose" is intended, first of all, for recognition of various multicomponent chemical systems. A necessary condition for this is improvement of discriminating ability of sensor arrays. However, possible ways for sensor array optimization in what concerns required selectivity of sensitive layers and parameterization of sensor response still remain not understood adequately. Another important problem is that of "extra" sensors with small information content. The reason is that such "extra" sensors introduce "additional information noise". Indeed, due to diversity and complexity of interactions between the multicomponent chemical systems and sensor elements, the data obtained for such systems are multivariate, so one cannot say in advance how important a given property is for identification of a certain analyte (or class of analytes). In this case, the problem of recognition from multivariate data gets first priority. In other words, it becomes necessary to optimize grouping from the viewpoint of the best correspondence of the results obtained to the final aim of recognition. 


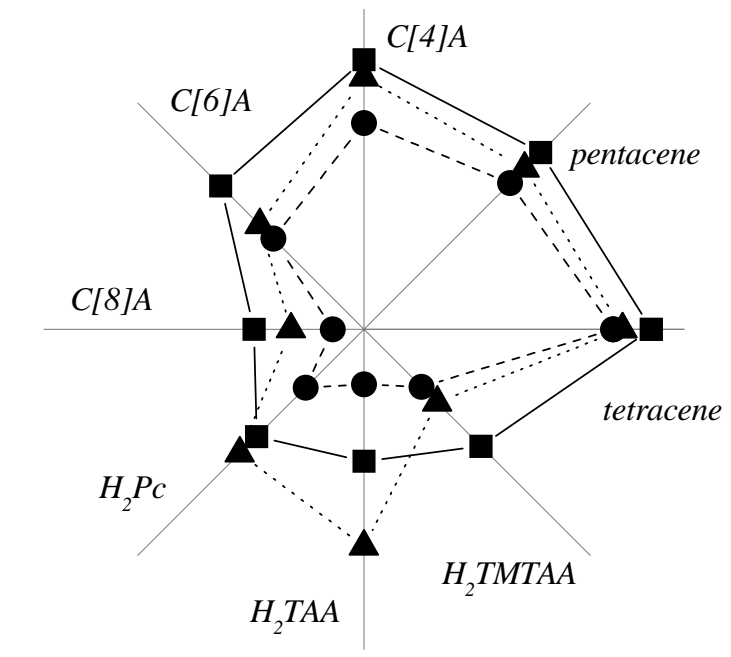

- - - . $\cdots$ first, second, third experiments

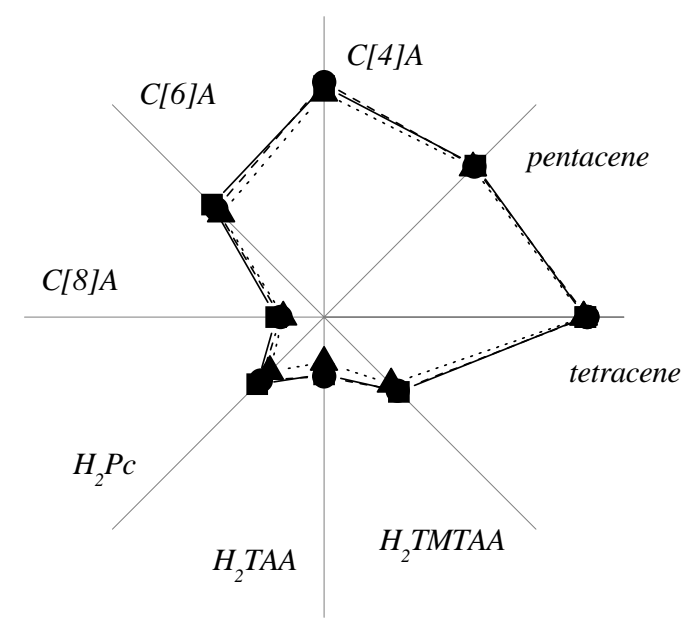

$-\mathbf{\square}-\cdots \mathbf{\Delta} \cdots$ first, second, third experiments

Fig. 3. Radial diagrams for three measurements of brandy samples without (a) and with (b) additional treatment of sensor surface.

\section{Results and discussion}

Quantitative analysis of recognizing ability of sensor array. To determine the possibilities for drinks recognition, we performed quantitative analysis of identification ability of sensor array. The cluster analysis methods that apply the fuzzy logic concept [13] were used as criterion. The input matrix involved the data of three experiments for each sample (for brandies and ethanol).

The key element of any optimization procedure is existence of recognition measure. It enables one to judge indirectly appropriateness of different sensors in a set and single out the most informative part of multidimensional response surface. The Rousseeuw version of cluster analysis makes it possible to perform such estimation using the so-called silhouette width $s(i)$ as parameter [13]. Being a discrimination degree, this parameter characterizes factually measurement association with a certain cluster. If the $s(i)$ values are close to unity, then the data are arranged compactly in a cluster, especially if all the $s(i)$ values are comparable to each other for all the cluster elements. Small values of $s(i)$ indicate that the corresponding measurement lies between the compact cluster regions. This means that, remaining within the framework of the fuzzy logic concept, one cannot conclude to what cluster the corresponding measurement belongs. Negative value of $s(i)$ indicates that, most probably, the corresponding measurement was erroneously associated with this cluster, i.e., it cannot be correctly associated with any of clusters formed from the data set under consideration. Minimization of the corresponding objective function for a certain combination of variables and observations makes it possible to calculate $s(i)$ for each observation, as well as its mean value $S(i)$ for the whole data set.

The $i V$-parameter (the normalized area below the kinetic curve) was calculated for various time intervals. A comparative analysis led to the conclusion that in this case application of that parameter enables one to improve considerably the degree of recognition for different types of samples [14]. Just such kind of sensor response parameterization was applied in our quantitative analysis.

The $i V$-parameters for complete set of sensors were used in different intervals of time: $0.7 \div 1.2,1.5 \div 5.2$, and $5.3 \div 7.8 \mathrm{~min}$. In the $0.7 \div 1.2 \mathrm{~min}$. interval, no sample was recognized. $(S(i)=0.45)$. In the case of $1.5 \div 5.2 \mathrm{~min}$. time interval, ethanol was recognized $(S(i)=0.33)$, while brandy $\mathrm{G}$ and ethanol were recognized in the $5.3 \div 7.8$ min. time interval $(S(i)=0.37)$. For further analysis, the $i V$-parameter at sampling times from 5.3 up to $7.8 \mathrm{~min}$. was chosen; in this case, the biggest $S(i)$ values $(S(i)=$ 0.37 ) were observed.

It is known that cluster analysis does not make it possible to choose optimal set of criteria (types of sensors) for solving the problem of drinks recognition. Therefore, the recognition ability was estimated for different combinations (sets) of sensors. An analysis of the results of recognition for normalized responses for five different samples (B, G, O, T, and ethanol) and different combinations (sets) of three sensors were made with $i V$-parameter for $5.3 \div 7.8 \mathrm{~min}$. time interval. This enabled us to estimate the effect of sensors on discrimination ability. It was shown that the best recognition occurs by sensors with pentacene, $\mathrm{C}[6] \mathrm{A}$, and $\mathrm{H}_{2}$ TAA coatings; two samples (brandy $\mathrm{G}$ and ethanol) are recognized in practically all cases. The sensor set used cannot recognize directly the brandies: $\mathrm{B}, \mathrm{O}$, and $\mathrm{T}$. Thus, for the samples with a dominant component, recognition ability is lower than that for other samples under consideration.

It is of interest to compare statistics of different sensors appearance in those three-sensor sets (formed of six combinations of C[4]A (2), C[6]A (3), pentacene (1), $\mathrm{H}_{2} \mathrm{Pc}(2), \mathrm{H}_{2}$ TMTAA (2), tetracene (2), C[8]A (1), and 
$\mathrm{H}_{2}$ TAA (4)) that give true recognition. In this case, one should note essential role of a sensor with $\mathrm{H}_{2}$ TAA coating (almost hydrophobic surface). The reason for this is that such drinks as brandy have heavy fractions with big organic molecules.

The sensor set under consideration could recognize directly and correctly only two objects of five. However, it is possible to develop a multistage approach. According to it, if a sample was assigned to a cluster that is common for several drinks, then further object recognition (within the cluster) is possible. Bearing this in mind, we analyzed the three unrecognized samples (brandies $\mathrm{B}, \mathrm{O}$, and $\mathrm{T}$ ) using different three-sensor sets (Table 1.). In this case, one can clusterize directly the brandy $\mathrm{B}$, and it is possible to separate it from two other brandy samples.

When studying statistics of appearance of different sensors in the three-sensor sets giving true recognition of three unclusterized samples, five combinations of $\mathrm{C}[4] \mathrm{A}$ (2), C[6]A (1), pentacene (4), $\mathrm{H}_{2} \mathrm{Pc}(0), \mathrm{H}_{2}$ TMTAA (5), tetracene (1), C[8]A (2), and $\mathrm{H}_{2}$ TAA (0) were considered. The optimal set involves pentacene, C[6]A, and $\mathrm{H}_{2}$ TMTAA. Thus, only two unrecognized drinks (brandies $\mathrm{O}$ and $\mathrm{T}$ ) remain after two stages of recognition.

Table 1. Chemical image recognition of three brandy samples ("Borysphen" - B, “Oleksandriys'kyi” - O and "Tavriya" - T) with three-sensor sets.

\begin{tabular}{|l|l|l|c|c|c|l|}
\hline \multicolumn{3}{|c|}{ Type of sensor } & \multicolumn{3}{c|}{$\begin{array}{c}\text { Sample } \\
\text { recognition }\end{array}$} \\
\cline { 4 - 7 } \multicolumn{2}{|l|}{} & $\mathrm{B}$ & $\mathrm{O}$ & $\mathrm{T}$ & $\mathrm{S}(i)$ \\
\hline tetracene & pentacene & $\mathrm{H}_{2}$ TMTAA & + & - & - & 0.4 \\
\hline pentacene & $\mathrm{C}[4] \mathrm{A}$ & $\mathrm{H}_{2}$ TMTAA & + & - & - & 0.42 \\
\hline pentacene & $\mathrm{C}[6] \mathrm{A}$ & $\mathrm{H}_{2}$ TMTAA & + & - & - & 0.5 \\
\hline pentacene & $\mathrm{C}[8] \mathrm{A}$ & $\mathrm{H}_{2}$ TMTAA & + & - & - & 0.38 \\
\hline C[4]A & $\mathrm{C}[8] \mathrm{A}$ & $\mathrm{H}_{2}$ TMTAA & + & - & - & 0.31 \\
\hline
\end{tabular}

Table 2. Chemical image recognition of two brandy samples ("Oleksandriys'kyi” - $O$ and "Tavriya" - T) with three-sensor sets.

\begin{tabular}{|l|l|l|c|c|c|}
\hline \multicolumn{3}{|c|}{ Type of sensor } & \multicolumn{2}{c|}{$\begin{array}{c}\text { Sample } \\
\text { recognition }\end{array}$} & \\
\cline { 3 - 6 } \multicolumn{2}{|l}{} & $\mathrm{O}$ & $\mathrm{T}$ & $S(i)$ \\
\hline tetracene & $\mathrm{C}[4] \mathrm{A}$ & $\mathrm{H}_{2}$ TAA & + & + & 0.33 \\
\hline tetracene & $\mathrm{C}[6] \mathrm{A}$ & $\mathrm{H}_{2}$ TAA & + & + & 0.36 \\
\hline $\mathrm{C}[6] \mathrm{A}$ & $\mathrm{H}_{2} \mathrm{Pc}$ & $\mathrm{H}_{2}$ TAA & + & + & 0.25 \\
\hline $\mathrm{C}[6] \mathrm{A}$ & $\mathrm{H}_{2} \mathrm{Pc}$ & $\mathrm{H}_{2}$ TMTAA & + & + & 0.25 \\
\hline $\mathrm{H}_{2} \mathrm{Pc}$ & $\mathrm{H}_{2}$ TAA & $\mathrm{H}_{2}$ TMTAA & + & + & 0.25 \\
\hline
\end{tabular}

The results of analysis of the two unrecognized brandy samples $(\mathrm{O}$ and $\mathrm{T})$ made using different threesensor sets (the $i V$-parameters for $5.3 \div 7.8 \mathrm{~min}$. time interval) are presented in Table 2 . One can see that the best recognition is achieved using sensors with tetracene, $\mathrm{C}[6] \mathrm{A}$, and $\mathrm{H}_{2}$ TAA coatings. In this case, one should consider five combinations of C[4]A (1), C[6]A (3), pentacene (0), $\mathrm{H}_{2} \mathrm{Pc}(3), \mathrm{H}_{2}$ TMTAA (2), tetracene (2), $\mathrm{C}[8] \mathrm{A}(0)$, and $\mathrm{H}_{2}$ TAA (3) when studying statistics of different sensors appearance in the three-sensor sets giving true recognition of two unclusterized samples.
Thus, the hierarchical clusterization procedure that is based, at each stage, on the fuzzy logic concept makes it possible, in the final analysis, to achieve complete recognition of all the samples under consideration.

\section{Conclusion}

Application of sensor cleaning procedure is proposed that not only ensures removal of organic molecules from the sensitive layer but makes it possible to restore the initial state of sensor surface as well. It is shown that treatment of sensor array with ethanol vapor can improve considerably system state restoration. This ensures formation of stable chemical images in the case of samples with dominant components. It is shown also that a two-stage clusterization procedure that is based, at each stage, on the fuzzy logic concept enables one to make complete recognition of all the samples considered.

\section{Acknowledgements}

The author is grateful to Dr. B. Snopok for helpful discussions.

\section{References}

1. K.J. Albert, N.S. Lewis, C.L. Schauer, G.A. Sotzing, S.E. Stitzel, T.P. Vaid, D.R. Walt, Cross-reactive chemical sensor arrays // Chem. Rev. 100, p. 2595 (2000).

2. B.A. Snopok, I.V. Kruglenko, Multisensor systems for chemical analysis: state-of-the-art in Electronic Nose technology and new trends in machine olfaction // Thin Solid Films 418(1), p. 21-41 (2002).

3. N. Paulsson, E. Larsson, F. Winquist, Extraction and selection of parameters for evaluation of breath alcohol measurement with an electronic nose // Sens. Actuators A: Phys. 84(3), p. 187-197 (2000).

4. L. Lvova, R. Paolesse, C. Di Natali, A. D'Amico, Detection of alcohols in beverages: An application of porphyrin-based Electronic Tongue // Sens. Actuators B: Chem. 118(1), p. 439-447 (2006).

5. T. Nakamoto, Y. Nakahira, H. Hiramatsu, T. Moriizumi, Odor recorder using active odor sensing system // Sens. Actuators B: Chem. 76(1), p. 465-469 (2001).

6. Y. Liu, X. Yu, R. Zhao, D.-H. Shangguan, Z. Bo, G. Liu, Quartz crystal biosensor for real-time monitoring of molecular recognition between protein and small molecular medicinal agents // Biosensors \& Bioelectronics 19(1), p. 9-19 (2003).

7. F. Jalali, E. Arkan, G. Bahrami, Preparation of a gabapentin potentiometric sensor and its application to pharmaceutical analysis // Sens. Actuators B: Chem., 127(1), p. 304-309 (2007).

8. R.M.A. Azzam, N.M. Bashara, Ellipsometry and Polarized Light, North-Holland, Amsterdam, 1980. 
9. J. Simon, J.-J. Andre, Molecular Semiconductors, Springer-Verlag, Berlin (1985).

10. Y.M. Shirshov, B.A. Snopok, O.V. Rengevych, V.I. Kalchenko, A. Coleman, Relaxation of nanostructured molecular materials under the influence of solvent vapors, in Frontiers of Multifunctional Nanosystems, Eds. E. Buzaneva, P. Scharff, p. 347-368, Kluwer Academic Publishers (2002).

11. I.V. Kruglenko, B.A. Snopok, Y.M. Shirshov, E.F. Venger, Digital aroma technology for chemical sensing: temporal chemical images of complex mixtures // SQO 3(4), p. 529-541 (2000).
12. I.V. Kruglenko, B.A. Snopok, Sensor renewal using displacement reactions, in $2^{\text {nd }}$ Workshop of the Second Network on Artificial Olfactory Sensing (NOSEII), New concepts for chemical sensing, Linköping University, Sweden, May 1821, 2003.

13. L. Kaufman, P.J. Rousseeuw, Finding Groups in Data: An Introduction to Cluster Analysis, Wiley, New York (1990).

P.J. Rousseeuw, Silhouettes: A graphical aid to the interpretation and validation of cluster analysis // J. Comput. Appl. Math. 20, p. 53-65 (1987). 